\title{
Der selbstoptimierende Fermenter basierend auf HPLC-Technologie
}

\author{
Andreas Ziegler ${ }^{1}$, Alexander Kron ${ }^{1}$, Sandra Dounia ${ }^{2}$, Daniel Sturm ${ }^{3}$, Johannes Bader ${ }^{2,4}$, Matthias \\ Rädle ${ }^{1}$ und Ulf Stahl ${ }^{2,4}$ \\ ${ }^{1}$ Hochschule Mannheim, Institut für Prozessmesstechnik und innovative Energiesysteme \\ ${ }^{2}$ Technische Universität Berlin, Fachgebiet für Angewandte und Molekulare Mikrobiologie \\ ${ }^{3}$ Wissenschaftliche Gerätebau Dr. Ing. Herbert Knauer GmbH, Berlin \\ ${ }^{4}$ Versuchs- und Lehranstalt für Brauerei in Berlin e.V., Forschungsinstitut für Mikrobiologie
}

\begin{abstract}
Das vorgestellte Projekt befasst sich mit der Entwicklung eines automatisch regulierten Bioreaktors. Dazu wurde eine UHPLC über ein eigens entwickeltes Probenahmesystem an einen Bioreaktor gekoppelt. Die erfassten Analysendaten werden zusammen mit den Messdaten wie $\mathrm{pH}$ und $\mathrm{pO}_{2}$ zur Interpretation und Steuerung des Fermentationsprozesses ausgewertet. Nach einer „Anlernphase“ kann der Fermenter somit eigenständig die Fütterungssteuerung anpassen bzw. Induktionsprozesse starten. Als Beispielapplikation für die Systementwicklung wurde Bacillus subtilis in Fed-Batch Kultivierungen gewählt. Unter ungünstigen Milieubedingungen oder hoher Zelldichte findet die Bildung von Endosporen als Überdauerungsform statt. Die Bildung der Endosporen soll mit dem entwickelten System kontrolliert und gezielt induziert werden.
\end{abstract}

\section{Einleitung}

Die bestehenden Kontrollsysteme für Bioreaktoren, basierend auf konventionellen Sensoren ( $\mathrm{pH}-, \mathrm{T}-, \mathrm{pO}_{2^{-}}$, etc.), geben keine ausreichende Information über den Verlauf komplexer biochemischer Reaktionen. Die gebildeten Zielprodukte sowie der Zellzustand stellen während der Fermentation häufig eine unbekannte Größe dar. Meist werden die fehlenden Informationen zeitversetzt durch Offline-Analytik beschafft. Zur Optimierung und Überwachung der Prozesse ist allerdings eine möglichst zeitnahe Quantifizierung relevanter Konzentrationen im Bioreaktor erforderlich [1][2]. Diese ist nur mit einer automatisierten Probenahme und moderner Analytik möglich. Der Einsatz automatisierter Probenahmesysteme in Kombination mit modernen UHPLC Systemen ermöglicht hierbei kurze Messintervalle und somit die Entwicklung neuer Regulierungs- und Optimierungsansätze. Als Modellorganismus für die Entwicklung wurde Bacillus subtilis herangezogen. Die Adaption des entwickelten Systems an andere Organismen und biotechnologische Produkte ist problemlos möglich.

\section{Probenahmemodul}

Für die Kopplung der UHPLC an den Bioreaktor muss das Probenahmemodul einen sterilen und partikelfreien Filtratstrom gewährleisten. Die Totvolumina innerhalb des Moduls sind zu minimieren, um eine schnelle und exakte Quantifizierung zu ermöglichen. Weiterhin muss das Probenahmemodul im Prozess autoklavierbar sein. Eine einfache Handhabung sowie der unterbrechungsfreie Einsatz im Dauerbetrieb am Bioreaktor müssen ebenfalls gewährleistet sein. Die Filtration wird über einen Querstromfilter realisiert.

\subsection{Konstruktion des Querstromfilters}

Die Entwicklung des Querstromfilters berücksichtigt sowohl die einfache Handhabung als auch die kostengünstige Fertigungsmöglichkeit. Der Querstromfilter besteht aus Lieferteilen sowie zwei PEEK-Drehteilen (siehe Bild 1). Er kann in wenigen Minuten demontiert, gereinigt und wieder montiert werden. Als Filtermembranen können kommerziell erhältliche Filter eingesetzt werden.

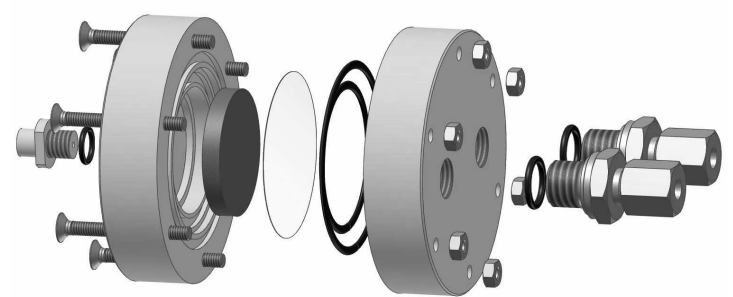

Bild 1 Explosionszeichnung des Querstromfilters 


\subsection{Simulation des Querstromfilters zur Probenahme}

Die optimierte Strömungsführung ermöglicht den kontinuierlichen Abtrag von Zellen und Feststoffen vom Filter zurück in den Bioreaktor und verhindert somit den Aufbau eines Filterkuchens. Um eine

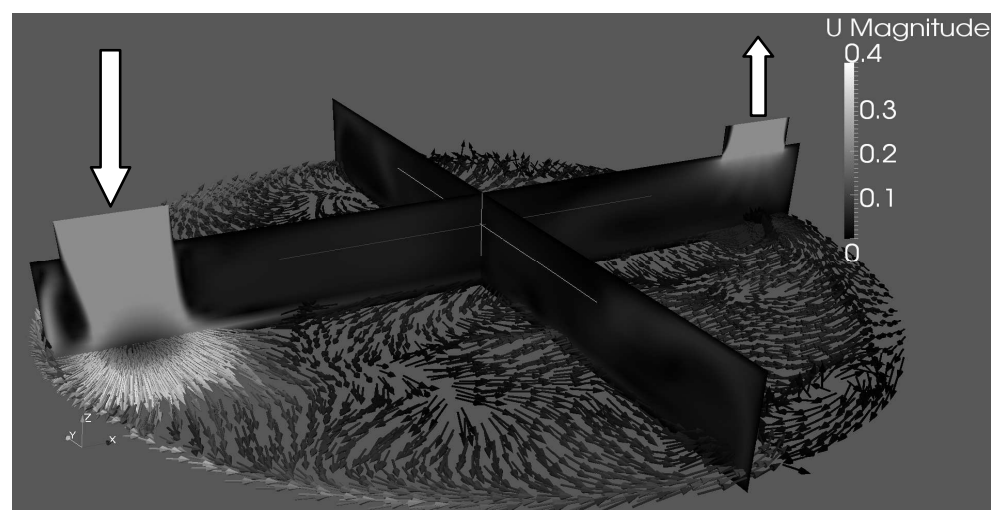

Bild 2 Strömungsprofil im Querstromfilter (berechnet durch OpenFOAM) geeignete Filtergeometrie $\mathrm{zu}$ ermitteln wurden mehrere Strömungssimulationen durchgeführt.

Das Strömungsprofil in Bild 2 zeigt eine gleichmäßige Überströmung der Filteroberfläche. Es kommt zu keinen Strömungskurzschlüssen zwischen Einund Auslass.

Die entstehenden Verwirbelungen sind zur Abtragung des Filterkuchens förderlich. Dies ist notwendig, um einen unterbrechungsfreien Dauerbetrieb $\mathrm{zu}$ gewährleisten.

\section{Selbstoptimierung}

Die Strategie der Selbstoptimierung orientiert sich an dem Ablauf evolutionärer Algorithmen [3]. Die komplexen, mehrdimensionalen Prozessführungsstrategien von Bioprozessen besitzen eine nahezu unendliche Variationsmöglichkeit. Daher ist es wichtig, von Beginn an möglichst viele Ausgangs Parametersätze zu besitzen. Die Integration von Anwenderwissen soll mehrere Iterationen der Optimierung ersparen. Da die Optimierungsstrategie nicht modellgestützt abläuft, ist die Anzahl der möglichen Iterationen auf die Anzahl der tatsächlich durchgeführten Fermentationen begrenzt.

In Bild 3 ist der Ablauf der Optimierung schematisch dargestellt. Zunächst wird eine Vielzahl von Prozessführungsstrategien durch den Anwender in das System eingepflegt. Diese werden von einem Interpreter in die Datenbank der Regelungsalgorithmen geschrieben. Jede dieser anwenderdefinierten Regelalgorithmen wird mehrmals zur Prozesssteuerung im Fermenter angewandt. Die hierbei während der Fermentation gewonnenen Prozessdaten werden einer Gütebewertung unterzogen.

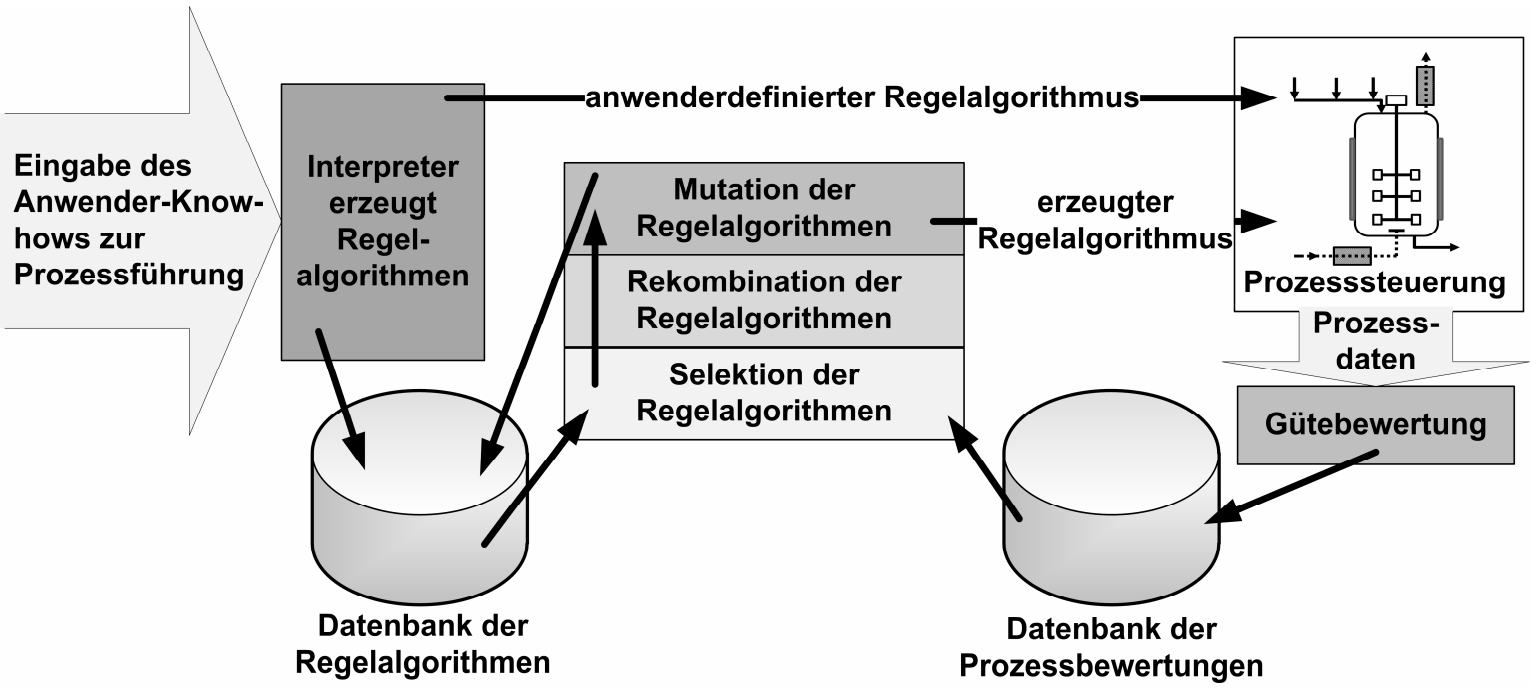

Bild 3 Systematik des selbstoptimierenden Fermenters mit evolutionärer Optimierungsstrategie

Die Gütebewertungen werden in der Datenbank der Prozessbewertungen abgelegt. Diese Bewertungen sind eindeutig den einzelnen Regelalgorithmen zuzuordnen. Ist eine ausreichende Anzahl an Prozessbewertungen vorhanden, werden die vielversprechenden Regelungsalgorithmen aus der Datenbank der Regelalgorithmen ausgewählt (Selektion der Regelalgorithmen). Diese werden schließlich parameterweise vermischt, um Nachkommen zu erzeugen (Rekombination der Regelalgorithmen). Die Parametersätze der Nachkommen 
werden schließlich noch in geringem Rahmen zufällig variiert (Mutation der Regelalgorithmen). Die erzeugten Regelalgorithmen werden schließlich ebenfalls zur Prozesssteuerung verwendet und einer Gütebewertung unterzogen. Zudem werden sie in der Datenbank der Regelalgorithmen abgelegt.

Die Güte der Prozessführung wird sich von Generation zu Generation unter leichter Fluktuation langsam auf ein Optimum zubewegen. Von entscheidender Bedeutung für die Geschwindigkeit und die Qualität der Optimierung sind der Umfang und die Varianz der anwenderdefinierten Reglungsstrategien. Liegen nur wenige Informationen zur Prozessführung vor, hat dies verschiedene Auswirkungen auf die Optimierung. Sind z.B. viele Prozessführungsstrategien vorgegeben, jedoch nur von geringer Güte, kann es zu Beginn der Optimierung noch zu starken Fluktuationen der Güte bis hin zu Totalausfällen kommen. Liegen hingegen nur wenige Prozessführungsstrategien vor, diese jedoch von hoher Güte, ist die Gefahr hoch, in einem lokalen Optimum stehen zu bleiben und das globale Optimum nicht zu erreichen. Sind Varianz und Güte der Anwendervorgaben jedoch ausreichend, wird die Optimierung das globale Optimum anstreben.

\section{Biotechnologische Ergebnisse}

Die Entwicklung des Kontroll- und Steuerungssystems erfolgte am Beispiel einer Kultivierung im Fed-Batch

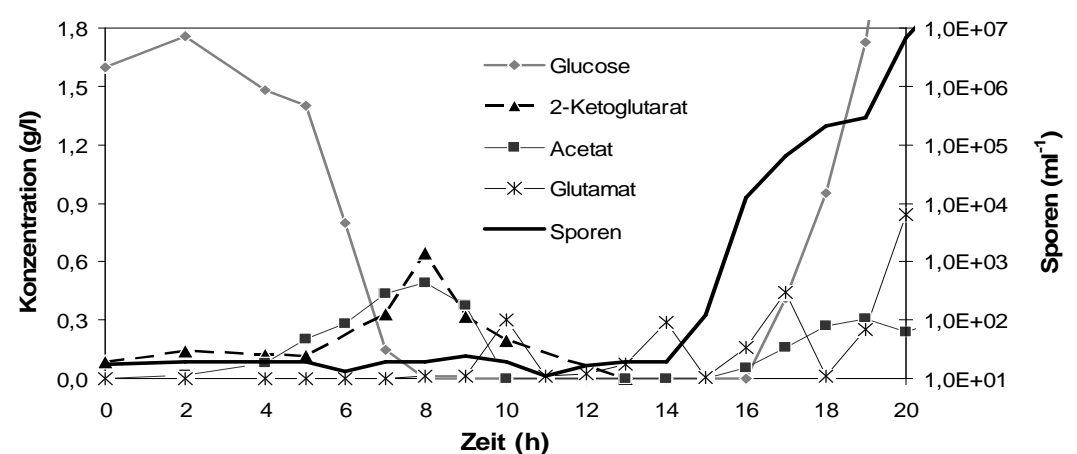

Bild 4 Fed-Batch Fermentation von B. subtilis (chemisch definiertes Medium, C-Quelle Glucose, Inokulum $10^{7}$ Zellen $/ \mathrm{ml}$, Belüftung 1vvm, $\mathrm{pO}_{2}$ geregelt über Rührerdrehzahl, $\mathrm{T}=30^{\circ} \mathrm{C}$, Fütterung $0-24 \mathrm{~h}$ mit $\mu=0,25 \mathrm{~h}^{-1}$
Verfahren mit Bacillus subtilis als Modelorganismus. B. subtilis ist dazu befähigt, unter ungünstigen Bedingungen wie beispielsweise Nährstoffmangel, hohe Zelldichte und andere Stress auslösende Faktoren Endosporen als Überdauerungsform zu bilden.

Bild 4 zeigt eine Fermentation unter Substratlimitierung. Mit dem Eintreten der Limitierung wird ein Maximum an 2-Ketoglutarat detektiert, was als Stellgröße für die Steuerung des Prozesses herangezogen werden kann. Für die Steuerung des Prozesses

bedeutet die Detektion des 2-Ketoglutarates beispielsweise, dass ein Ereignis ausgelöst wird, welches die Kultur entweder durch ein angepasstes Fütterungsprofil im vegetativen Status hält oder aber durch Zugabe einer entsprechenden Substanz [4] die initiierte Sporulation stimuliert.

Die Sporulation umfasst mehrere Stadien morphologischer Veränderungen [5] und ist nur in einem kleinen Zeitfenster reversibel [6]. Der Zeitraum, in dem der Sporulation entgegengewirkt werden kann, ist für die Steuerung entscheidend, wenn der Focus bei der Fermentation von Bacillus auf der Biomasse liegt.

Durch fraktionierte Einzelgaben von Glucose $(4 \mathrm{~g} / 1)$ konnte in einer kontrolliert geführten Fermentation die bestehende Sporenkonzentration konstant gehalten und ein Anstieg um sechs Stunden verzögert werden. Einen Vergleich der kontrolliert geführten FedBatch Fermentation mit deren Referenz zeigt Bild 5.

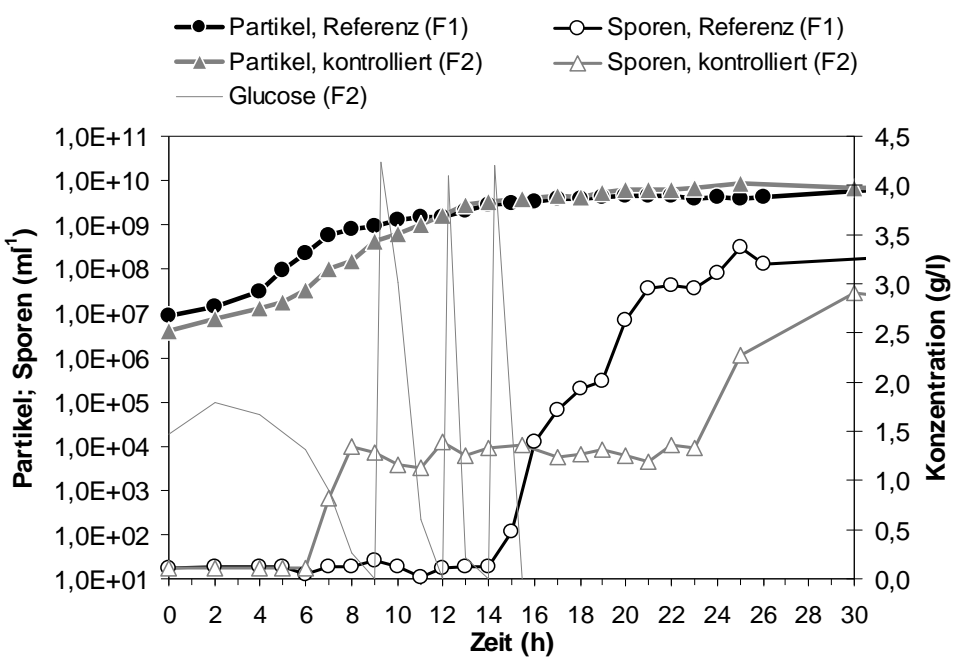

Bild 5 Sporen- und Zellzahl von Referenz- und kontrollierter FedBatch Fermentation im Vergleich (in Bild 5 beschriebene Fermentation diente als Referenz; kontrollierte Fermentation unter identischen Bedingungen. Zugabe der Substratfraktionen nach 9h, 12h und 14h) 


\section{Zusammenfassung}

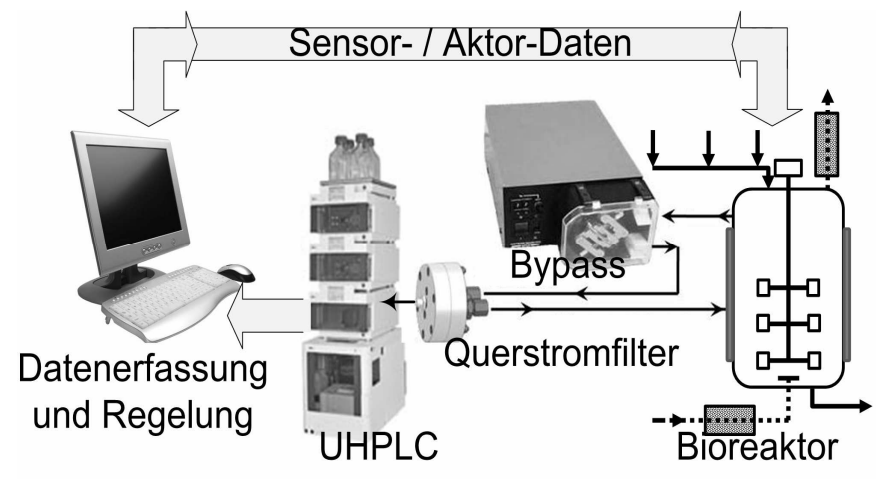

Bild 6 Schematischer Systemaufbau des selbstoptimierenden Fermenters mit Probenahme und UHPLC-Analytik
Im verwendeten Systemaufbau (siehe Bild 6) wird eine UHPLC (KNAUER GmbH, Deutschand) in Kombination mit einem auf Cross-Flow basierenden Probenahmemodul zur Analyse verwendet. Das Filtermodul wurde dabei so konstruiert, dass ein unterbrechungsfreier Betrieb über $240 \mathrm{~h}$ möglich ist. Bereits während der laufenden Messung der UHPLC wird die Probenschleife neu von Filtrat durchspült und somit eine sehr schnelle Analysenfolge ermöglicht. Die erhaltenen Messergebnisse werden automatisch ausgewertet und für die Steuerung der Fütterungsstrategie herangezogen.

\section{Ausblick}

Die Online-Analytik bestimmter Metabolite, Substrate und Endprodukte basierend auf UHPLC-Technologie bietet die Möglichkeit, die Optimierung und Überwachung von Bioprozessen zu verbessern. Die Dokumentation von Daten in Echtzeit ermöglicht neue und komplexe Überwachungsstrategien für verschiedenste Bioprodukte. Aufgrund der gewonnenen Erkenntnisse im Rahmen des Projektes sowie der ständig wachsenden Nachfrage nach optimierten Verfahren und Prozessen für die industrielle Biotechnologie sind die beteiligten Partner bestrebt, die Entwicklung im Bereich selbstoptimierender Bioreaktoren konsequent voranzutreiben.

\section{Danksagungen}

Der Sartorius Stedim Biotech $\mathbf{G m b H}$ danken wir für die Bereitstellung von BioPAT ${ }^{\circledR}$ MFCS / win 3.0, Recipe Control (S88)-modules for BioPAT ${ }^{\circledR}$ MFCS / win 3.0 und BioPAT ® MFCS / win OPC DA-Server. Durch diese gewährte Unterstützung ist es möglich, eine stabile und einfache Kommunikation zwischen dem Optimierungssystem und dem Bioreaktor herzustellen.

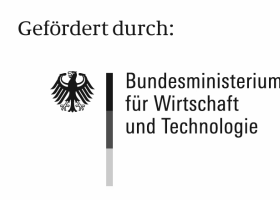

aufgrund eines Beschlusses des Deutschen Bundestages

\section{Literatur}

[1] B. Hitzmann, T. Scheper: Bioprozesstechnik, Bioprozessanalytik und -steuerung. Spektrum Akademischer Verlag 2011

[2] S. Büttgenbach, M. Michalzik, R. Wilke: Neue Ansätze zur Online-Bioprozessanalytik. Chemie Ingenieur Technik, 77, S. 833-839. Weinheim: WILEY-VCH Verlag 2005.

[3] Volker Nissen: Einführung in Evolutionäre Algorithmen. Wiesbaden: Verlag Vieweg 1997.

[4] J.H. Hageman, G.W. Shankweiler, P.R. Wall, K. Franich, G.W. McCowan, S.M. Cauble, J. Grajeda,

C. Quinones: Single, Chemically Defined Sporulation Medium for Bacillus subtilis: Growth, Sporulation, and Extracellular Protease Production. Journal of Bacteriology, S. 438-441, 1984

[5] P. Stragier, R. Losick: Molecular genetics of sporulation in Bacillus subtilis. Annu. Rev. Genet., 30, S 297-341, 1996

[6] S. Kretschmer: Reversion sporulierender Bacillus megaterium-Zellen zum Wachstum. Zeitschrift für allg. Mikrobiologie, 12, S. 459-467, 1972 